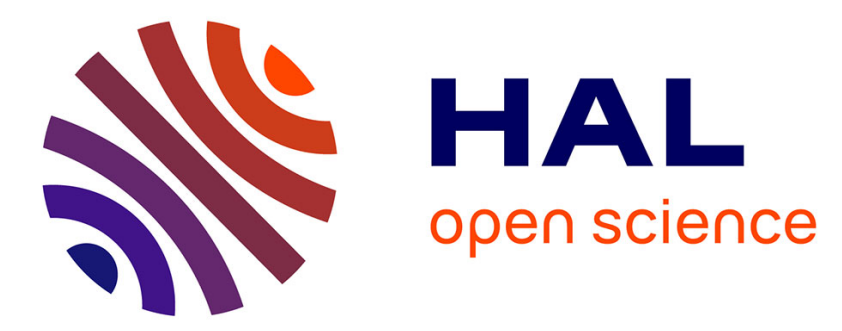

\title{
The Structure of the $\alpha$-quartz (0001) Surface Investigated Using Helium Atom Scattering and Atomic Force Microscopy
}

\author{
W. Steurer, A. Apfolter, M. Koch, Thomas Sarlat, E. Sondergard, W.E. \\ Ernst, B. Holst
}

\section{To cite this version:}

W. Steurer, A. Apfolter, M. Koch, Thomas Sarlat, E. Sondergard, et al.. The Structure of the $\alpha$-quartz (0001) Surface Investigated Using Helium Atom Scattering and Atomic Force Microscopy. 24th European Conference on Surface Science (ECOSS-24), Sep 2006, Paris, France. pp.4407-4411. hal-00504494

\section{HAL Id: hal-00504494 \\ https://hal.science/hal-00504494}

Submitted on $20 \mathrm{Jul} 2010$

HAL is a multi-disciplinary open access archive for the deposit and dissemination of scientific research documents, whether they are published or not. The documents may come from teaching and research institutions in France or abroad, or from public or private research centers.
L'archive ouverte pluridisciplinaire HAL, est destinée au dépôt et à la diffusion de documents scientifiques de niveau recherche, publiés ou non, émanant des établissements d'enseignement et de recherche français ou étrangers, des laboratoires publics ou privés. 


\title{
The Structure of the $\alpha$-quartz (0001) Surface Investigated Using Helium Atom Scattering and Atomic Force Microscopy
}

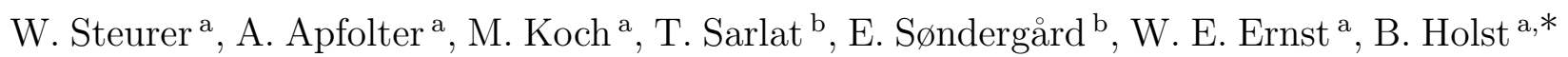 \\ ${ }^{a}$ Graz University of Technology, Institute of Experimental Physics, Petersgasse 16, 8010 Graz, Austria \\ ${ }^{\mathrm{b}}$ Laboratoire Surface et Interface du Verre, UMR125CNRS/Saint-Gobain, 39 Quai Lucien Lefranc, 93303 Aubervilliers Cedex, France
}

\begin{abstract}
In this paper we present the first structural investigation of quartz using helium atom scattering (HAS). Studies were carried out on the $\alpha$-quartz (0001) surface (Z-cut). He-diffraction experiments reveal a complex hexagonal diffraction pattern. Prominent peaks are visible at positions corresponding to the periodicity of the bulk unit cell $(4.90 \pm 0.02 \AA)$. In addition weak $(2 \times 2)$ peaks and satellite peaks are observed. Two recent theoretical papers both propose a $(1 \times 1)$ reconstruction for $\alpha$-quartz (0001) with $120^{\circ}$ symmetry for the surface unit cell: the so called dense structure. We propose a model where terraces with step heights of a multiple $1 / 3$ of the unit cell height lead to domains rotated by $\pm 60^{\circ}$ relative to each other. This is supported by AFM images. The satellite peaks indicate an incommensurate super-lattice structure with a periodicity of the order of $57 \AA$, presumably related to strain relief mechanisms. The $(2 \times 2)$ peaks may stem from co-existing regions of a semi-dense structure, which is predicted to have a higher energy than the dense, $(1 \times 1)$ phase.
\end{abstract}

Key words: helium atom scattering, AFM, quartz surface structure PACS: $61.18 . \mathrm{Bn}$

\section{Introduction}

Although quartz surfaces are extremely important substrates in many fields spanning from molecular biology [1] to micro technologies [2], and although quartz is produced industrially on a large scale [3] surprisingly little fundamental work on the surface structure has been carried out up till now. Possibly this is partly due to the fact that quartz is an insulating material and hence difficult to investigate with many surface science techniques.

Helium atom scattering (HAS) where neutral, low energy, ground state atoms are used as a probe [4] and atomic force microscopy (AFM) are particularly suited for the investigation of insulating surfaces. In this paper in-plane HAS has been used to characterize the $\alpha$-quartz (0001) surface structure on the atomic scale, whereas the AFM provides an overall characterization of the surface topography on the nanometer scale.

After a discussion of the work carried out on the $\alpha$ quartz (0001) surface up till now, the paper moves on in Section 2 to discuss the experimental setup and the sample

\footnotetext{
* Corresponding author.

Email address: bodil@cantab.net (B. Holst).
}

preparation procedure for the experiments presented here. The results are presented in Section 3. Then a discussion follows of the proposed surface structure in Section 4 and finally a conclusion is presented in Section 5 .

AFM images of synthetically grown $\alpha$-quartz (0001) surfaces were obtained by Kavasaki et al. [5]. The focus of [5] is on growth mechanisms and the images show the crystal facets without any polishing or any further surface treatment. The unpolished (0001) surface displays a so called cobbles structure with two types of "hills" with characteristic heights of 20-50 $\mu \mathrm{m}$ and up to $480 \mu \mathrm{m}$.

The first low energy electron diffraction (LEED) experiments on the $\alpha$-quartz (0001) surface were reported by Janossy and Menyhard [6]. The crystal was polished and then etched in HF to remove damaged layers. No further cleaning process was carried out. The observed LEED pattern indicated a $(1 \times 1)$ surface structure, which disappeared upon heating to $500{ }^{\circ} \mathrm{C}$. More detailed LEED studies were carried out by Bart and Gautier [7]. After mechanical polishing, the samples were chemically etched in a $2 \% \mathrm{HF}$ solution for several minutes and then annealed in air over several hours at various temperatures up to $1200{ }^{\circ} \mathrm{C}$. Samples annealed up to $500{ }^{\circ} \mathrm{C}$ showed the expected $(1 \times 1)$ pattern. Annealing to a temperature above $600{ }^{\circ} \mathrm{C}$ in air 
leads to a reconstructed LEED pattern $\left.(\sqrt{84} \times \sqrt{84}) R 11^{\circ}\right)$. Bulk $\alpha$-quartz shows a periodic defect structure called the Dauphiné twins [8]. Bart and Gautier suggested that this defect type is linked to the observed surface reconstruction.

Recent simulations of the $\alpha$-quartz (0001) surface suggest that the surface undergoes a reconstruction leading to a $(1 \times 1)$ structure $[9,10]$. This will be discussed in details in Section 4.

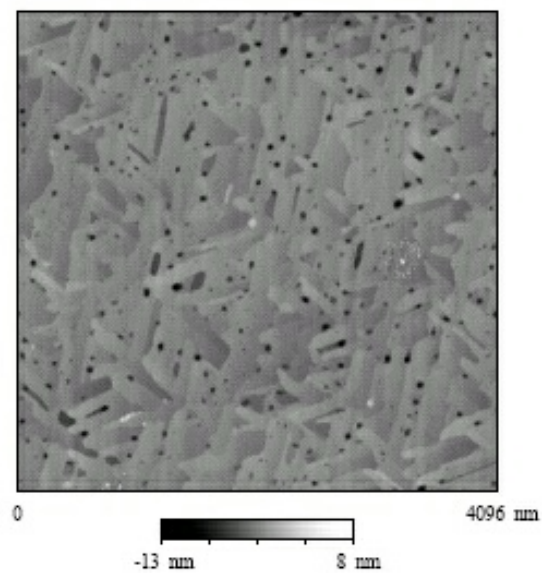

Fig. 1. AFM image of the $\alpha$-quartz (0001) surface. The image shows large, atomically flat terraces with an average width of about $200 \mathrm{~nm}$ and an average step height of around $1.9 \mathrm{~nm}$. A characteristic feature of the surface is that the terraces are oriented with angles of $60^{\circ}$ relative to each other. Furthermore the surface shows a range of pits with depths between 2 and $5 \mathrm{~nm}$, presumably created through relaxation processes during the annealing.

\section{Experiment}

A synthetically grown, Z cut (0001) (miscut less than $\left.0.5^{\circ}\right)$, twin free, $10 \times 10 \times 1 \mathrm{~mm}^{3} \alpha$-quartz crystal polished to optical quality using diamond powder, was used in the present experiments. Two samples cut from the same mother crystal were investigated using AFM and LEED. One of the samples was investigated using HAS. The AFM image and HAS-data presented here are all from the same sample. The samples were cleaned in a soap solution (Alcanox) and then in three batches of deionized water, before finally annealing to $1025{ }^{\circ} \mathrm{C}$ for 72 hours under a pressure of 2 bar of oxygen. No etching was carried out. AFM images were obtained on a IIIA Nanoscope, Digital Instruments Inc. using $40 \mathrm{~N} \mathrm{Si}_{3} \mathrm{~N}_{4}$ cantilevers from Nanosensors. One of the crystals was transferred into a vacuum chamber (base pressure around $10^{-10}$ mbar) via a load lock with an oil free pumping system. The vacuum chamber was equipped with X-ray Photoemission Spectroscopy (XPS) and LEED. Despite the high annealing temperature the LEED pattern showed a clear $(1 \times 1)$ structure. This can perhaps be attributed to the high pressure (2 bar) and pure oxygen atmosphere during the annealing. Other LEED experiments (discussed in the introduction) were carried out after annealing in air. The carbon contamination of the surface was analyzed in vacuum using XPS. The contamination was removed by heating the crystal slowly (over about 3 hours) to $350{ }^{\circ} \mathrm{C}$ and bleeding in oxygen in the chamber at a high local partial pressure (about $10^{-4}$ ) mbar. The complete cleaning system and procedure is described extensively elsewhere [11]. When no more carbon could be traced at the surface the sample was transferred to a transport container in an argon atmosphere.

The sample was then transported to Graz for HAS experiments using the apparatus MAGIE [12]. The total transfer time between Paris and Graz was about 24 hours. In Graz the container was opened after it had been inserted in a flexible plastic glove box, filled with a slight overpressure of argon and mounted in a sealed manner onto a flange on the central chamber of MAGIE. In this way it was possible to mount the sample on the MAGIE sample holder and transfer it to the apparatus keeping it permanently under an argon atmosphere. The sample was placed on a molybdenum plate and kept in place by two stainless steel springs. To make the sample transfer process as quick as possible a Ktype thermocouple was placed, not on the sample surface, but pre-mounted (spot welded) next to the sample on the molybdenum plate. After the experiments had been completed, this thermocouple was calibrated by venting the chamber, placing an additional thermocouple on top of the sample and repeating all heating and cooling procedures. The sample holder itself was mounted on a six-axis manipulator. After the sample had been mounted the chamber was pumped down to about 1 mbar using a membrane pump and further pumped down by slowly opening a valve to a running turbo-pump. This procedure was adopted to prevent rotary pump oil from back streaming and contaminate the sample. The experiments were all carried out with a base pressure in the $10^{-9}$ mbar range. This pressure was obtained within two days without baking the chamber. Before starting experiments the surface was exposed to an initial cleaning in situ similar to the one described above and in [11]. The surface was found to be extremely inert against contamination, maintaining a constant He-reflectivity at room temperature over a period of days without any further cleaning.

For the HAS experiments an almost monochromatic beam was created by supersonic expansion through a nozzle of $10 \mu \mathrm{m}$ diameter. The central part of the beam was selected by a skimmer of $400 \mu \mathrm{m}$ diameter. The beam energy was $67.3 \mathrm{meV}$ with a spread of $\delta E / E \approx 2 \%$ for all experiments, monitored by a time-of-flight measurement (TOF) either directly before or after a diffraction experiment. The scattered helium atoms were ionized by electron bombardment, sent through a magnetic mass selector and detected by a channeltron. The detector entrance was $1618 \mathrm{~mm}$ from the sample surface. For the experiments presented here the scattering angle was kept fixed at $90^{\circ}$ and the crystal was rotated in the scattering plane between $10^{\circ}$ and $80^{\circ}$ in steps of $0.05^{\circ}$ and between $0^{\circ}$ and $90^{\circ}$ in the crystal plane (azimuthal angle) in steps of $30^{\circ}$, see Fig. 2. All experiments were carried out with the sample at room 


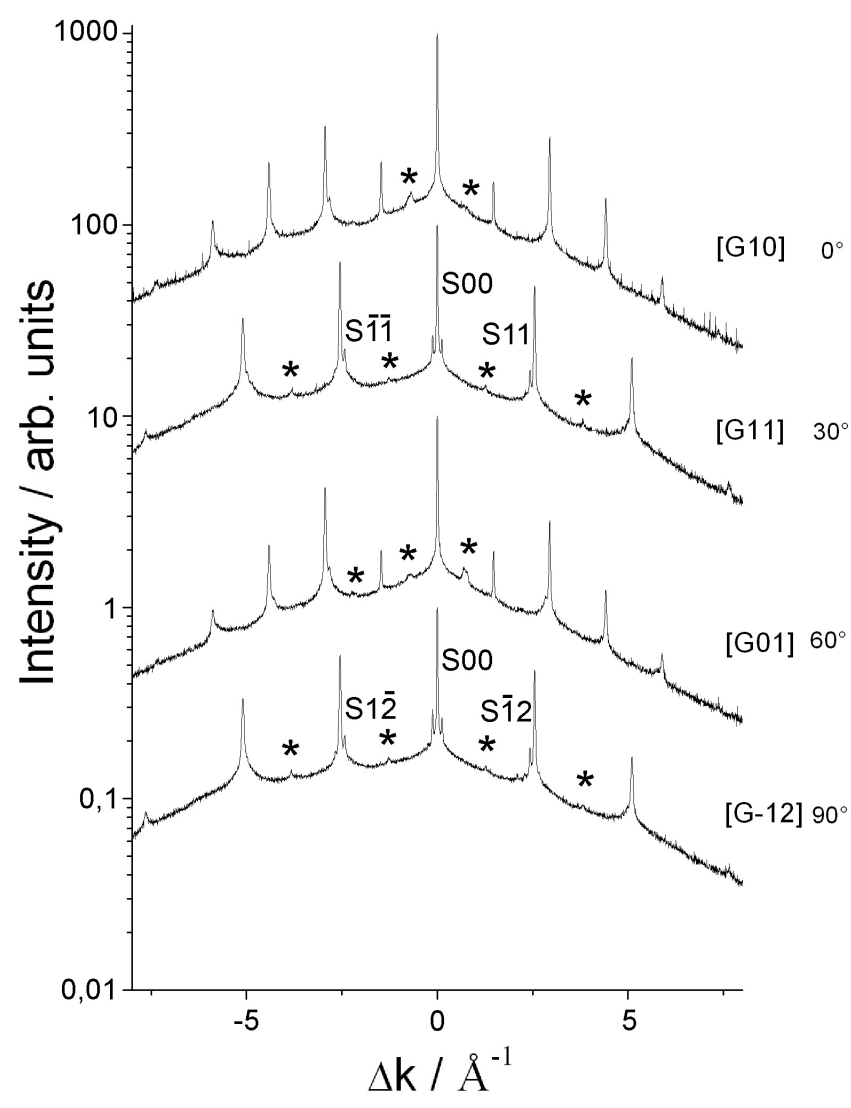

Fig. 2. In plane helium atom diffraction spectra for a range of crystal orientations (indicated on the right-hand side of the curves by the reciprocal lattice indices and the in plane angle, respectively). All spectra were obtained at a constant beam energy of $67.3 \mathrm{meV}$. SXXs denote the most prominent satellite peaks where $X X$ stands for the lattice indices of the related main peak. Additional weak peaks are labeled with an asterisk. The spectra are shifted by a multiplication of 10 from one graph to the next.

temperature.

\section{Results}

The AFM image (Fig. 1) shows large, atomically flat terraces/steps with an average width of about $200 \mathrm{~nm}$ and an average step height of around $1.9 \mathrm{~nm}$. A characteristic feature of the surface is that the terraces are oriented with angles of $60^{\circ}$ relative to each other. Furthermore the surface shows a range of pits with depths between 2 and $5 \mathrm{~nm}$, presumably created through relaxation processes during the annealing.

All HAS diffraction spectra (Fig. 2) have narrow specular peaks (about $0.006 \AA^{-1}$, FWHM, limited by the present angular resolution of the apparatus), indicating long terraces with a mean width of at least $200 \stackrel{\AA}{A}$ [13]. This is consistent with the AFM image. The high diffuse background indicates the presence of many defects (i.e. the pits visible

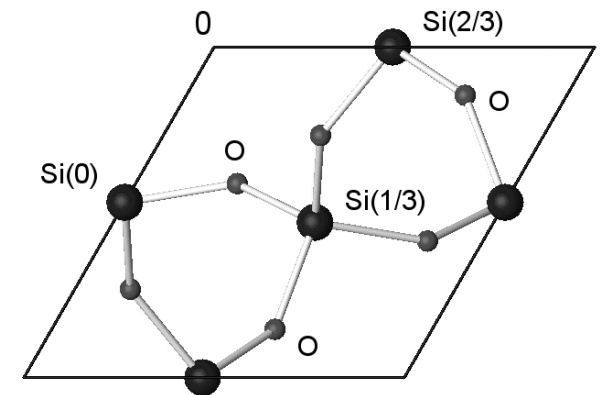

Fig. 3. This figure shows the $\alpha$-quartz bulk unit cell projected onto the (0001) plane. The large balls represent Si-atoms. The numbers in brackets indicate the relative positions of the Si-atoms in the bulk unit cell. The small balls represent oxygen atoms. Note that the $\operatorname{Si}(0)$ and $\mathrm{Si}(-2 / 3)$ atoms are not really connected, the impression occurs through the projection. The bulk structure of quartz can be seen as linked spirals of tetrahedrons (containing one silicon and four oxygen atoms). In an un-twinned sample the spirals all rotate in the same direction with three tetrahedrons making one full rotation. Each bulk unit cell contains segments of two spirals. The axis of rotation is perpendicular to the (0001) plane.

in the AFM image). The slight asymmetry of the diffraction pattern is attributed to the change in the beam footprint on the surface as the incident angle is varied.

The overall diffraction pattern is hexagonal. The periodicity of the most prominent peaks corresponds to a surface unit cell with parameters $a 1=a 2=4.90 \pm 0.02 \stackrel{\circ}{A}$, which are exactly the values for the bulk unit cell [14]. Additional weak peaks can be seen half way between the main peaks in both main directions (labeled by $\left(^{*}\right)$ in Fig. 2). In addition satellite peaks can be observed, most prominently in the $\mathrm{G}[11]$ and $\mathrm{G}[-12]$ directions (reciprocal lattice): $\Delta k=$ $0.13 \AA^{-1}$, calculated for the satellite peaks labeled in Fig. 2. The satellite peaks are asymmetric relative to the higher order diffraction peaks, shifted towards smaller momentum transfer.

\section{The Surface Structure}

Two groups have proposed similar models for the surface reconstruction of $\alpha$-quartz (0001). De Leeuw et al. [9] call it the bridge structure whereas Rignanese et al. [10] refer to it as the dense structure which is the labeling that we use in this paper.

The unit cell as it is found in the bulk is shown in Fig. 3 projected onto the (0001) plane. The bulk structure of quartz can be seen as linked spirals of tetrahedrons (containing one silicon and four oxygen atoms). In an un-twinned sample the spirals all rotate in the same direction with three tetrahedrons making one full rotation. Each bulk unit cell contains segments of two spirals as can be seen in Fig. 3. The axis of rotation of the spirals is perpendicular to the (0001) plane. The surface reconstruction labeled the dense structure can be understood as follows: Starting from the cleaved surface the topmost 
atoms are oxygen atoms with one dangling bond ${ }^{1}$ (See Fig. 4(a)). These oxygen atoms are connected to the topmost Si-atoms (large black balls in Fig. 4(a)), which are three coordinated. In the dense structure (see Fig.4(b)) a top oxygen atom of a cleaved surface connects to a neighbouring Si-atom lying two layers deeper $(-2 / 3)$, (pale grey in Fig. 4(a)). Since this Si-atom is already fully connected and since an over-coordinated atom is energetically not favorable, the topmost $\mathrm{Si}-\mathrm{O}-\mathrm{Si}$ bond which bridges to a Si-atom in the $(-1 / 3)$ layer (dark grey in Fig. 4(a)) will swap from the now over-coordinated $(-2 / 3)$ Si-atom to the under-coordinated top one. The top Si-atom and the Si-atom previously in the $(-1 / 3)$ layer relax to be at the same level. All silicon atoms are now four-coordinated and all oxygen atoms two-coordinated. The first atomic plane of the reconstructed surface now contains three times as many oxygen atoms and the Si-layer below twice as many Si-atoms as in the cleaved surface case (hence the labeling "dense structure"). The repeat distance for the reconstructed surface unit cell is that of the bulk. Neither de Leeuw et al. nor Rignanese et al. provide exact positions for the atoms at the reconstructed surface. Their calculations of the surface energy for the dense structure surface differ even though they propose the same structure: $\left(0.05 \mathrm{eV} / \AA^{2}\right)[10]$ and $0.12 \mathrm{eV} / \AA^{2}$ [9], respectively. The energy of the cleaved structure was calculated by Rignanese et al. [10] only to be $0.25 \mathrm{eV} / \stackrel{\circ}{ }^{2}$.

The diffraction patterns depicted in Fig. 4(a))show a $60^{\circ}$ symmetry and have the periodicity of the bulk lattice (ignoring the very small $(2 \times 2)$ peaks which will be discussed later $)$. The periodicity fits the $(1 \times 1)$ model proposed, but on the basis of the structure within the unit cell, one would expect $120^{\circ}$ symmetry of the intensity modulation rather than $60^{\circ}$. We propose a model, where the presence of terraces with step heights of a multiple $1 / 3$ of the unit cell height leads to domains rotated by $\pm 60^{\circ}$ relative to each other (see Fig. 5).

The AFM image indicates the presence of steps of various heights on the surface, which supports our model. However, the experimental resolution was not high enough to resolve with certainty step height differences close to one third of the unit cell height $5.405 \AA$ [15]. A strong supporting argument for our model is the frequent occurrence in the AFM image of steps with a $60^{\circ}$ angle with respect to the direction of the main steps of the surface.

We still have not explained the weak additional $(2 \times 2)$ peaks. The paper by Rignanese et al. [10] provides a possible explanation. This paper proposes four possible surface reconstructions. The dense $(1 \times 1)$ structure is the energetically most favorable with a surface energy of $0.05 \mathrm{eV} / \stackrel{\circ}{A}^{2}$ as mentioned earlier, but a proposed semi-dense surface structure with a $(2 \times 1)$ reconstruction has a surface energy

\footnotetext{
1 The surface could also terminate with Si-atoms, however, since natural silicon dioxide surfaces are generally assumed to terminate with hydroxil $(\mathrm{OH})$ groups, the oxygen-terminated surface seems to be more representative for real conditions. See [10].
}

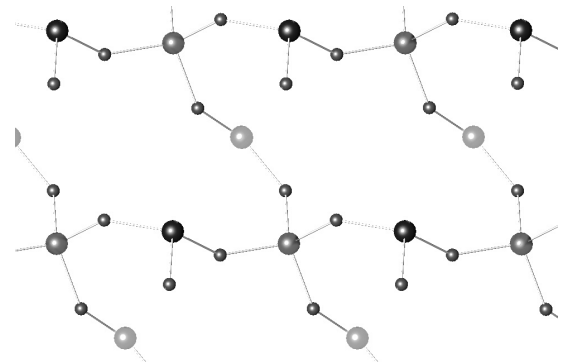

(a) cleaved surface

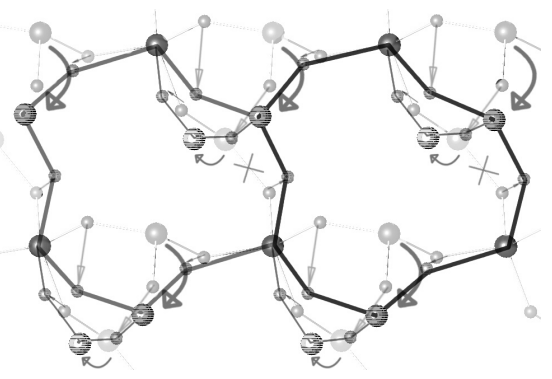

(b) dense structure

Fig. 4. Here the predicted reconstruction of the $\alpha$-quartz (0001) surface is illustrated. The big balls represent Silicon atoms, the little balls oxygen atoms. (a) This figure shows the cleaved surface: The grey scale indicates the vertical position of the Si-atoms (black on top at 0 , grey at $-1 / 3$ and pale grey at $-2 / 3$ bulk unit cell height). The top most Si-atoms (black) are three-coordinated and the top most oxygen atoms one-coordinated as illustrated. The Si-atoms at $-2 / 3$ height (pale grey) have two additional bonds (not shown) going to a layer deeper. (b) This figure sketches the reconstruction. In the background the cleaved surface and arrows are shown to illustrate how the reconstruction is formed. NB! the color code from (a) no longer applies. A one-coordinated oxygen atom connects to a neighbouring Si-atoms lying two layers deeper $(-2 / 3)$. The topmost $\mathrm{Si}-\mathrm{O}-\mathrm{Si}$ bond which bridges to a $\mathrm{Si}$-atom in the $(-1 / 3)$ layer swaps from the now over-coordinated $(-2 / 3)$ Si-atom to the under-coordinated top one (the bond breaking is indicated by $\times$ ). The bold lines indicate the new surface unit cell with $120^{\circ}$ symmetry.

of only $0.09 \mathrm{eV} / \AA^{2}$, quite close to the dense surface and still considerably below the energy of the cleaved surface $\left(0.25 \mathrm{eV} / \AA^{2}\right)$. It is conceivable that a small fraction of the surface shows a semi dense like reconstruction. This would explain the diffraction pattern since a hexagonal $(1 \times 1)$ structure combined with three domains of $(2 \times 1)$ can give a diffraction pattern similar to a $(1 \times 1)$ structure combined with a $(2 \times 2)$ structure $)$.

The satellite peaks must be subject to further investigation before anything conclusive can be said. They indicate an incommensurate super-lattice structure with a periodicity of the order of $57.5 \pm 1.5 \AA$, presumably related to strain relief mechanisms.

\section{Conclusion}

In this paper we present the first HAS diffraction data from a quartz crystal ( $\alpha$-quartz (0001)). The data shows a basic $(1 \times 1)$ structure. We propose a model of the $\alpha$ - 


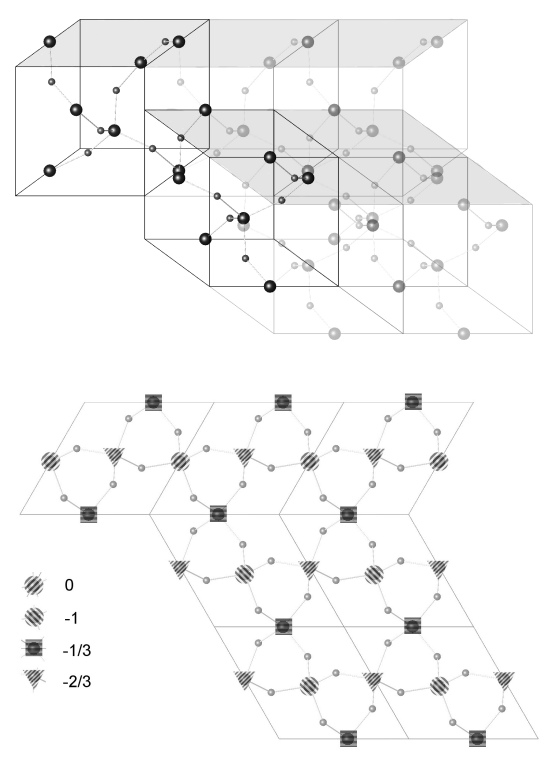

Fig. 5. A proposed model for the $\alpha$-quartz (0001) surface. Steps $1 / 3$ of the unit cell height, leads to domains rotated $\pm 60^{\circ}$ relative to each other. Note the spiral arrangement of the silicon atoms in the bulk. quartz (0001) surface where the presence of terraces with step heights $1 / 3$ of the unit cell height leads to domains rotated by $\pm 60^{\circ}$ relative to each other. This model and the experimental data agree well with predictions from theory which foresee a $(1 \times 1)$ surface reconstruction with $120^{\circ}$ symmetry of the surface unit cell. The occurrence of additional weak peaks can be explained as a $(2 \times 1)$ surface reconstruction with a slightly higher energy, also predicted by theory. The occurrence of satellite peaks around the main diffraction peaks is presumably due to an incommensurate super-lattice structure with a periodicity of the order of $57.5 \pm 1.5 \stackrel{\circ}{A}$.

\section{Acknowledgement}

We thank Daniel Farías and Giangelo Bracco for stimulating discussions. We thank Stephen C. Parker for comments on the bridge structure. This work was supported by the European Commission, FP6, NEST STREP ADVENTURE program, Project INA.

\section{References}

[1] Y. C. Sasaki, Biochem. Soc. Trans. 32, 761 (2004)

[2] P. Papakonstantinou, N. A. Vainos, and C. Fotakis, Appl. Surf. Sci. 151, 159 (1999)

[3] U.S. Geological Survey, Min. Comm. Summ.(1997)

[4] D. Farías and K. H. Rieder, Rep. Prog. Phys. 61, 1575 (1998)

[5] M. Kawasaki, K. Onuma, and I. Sunagawa, J. Cryst. Gr. 258, $188(2003)$

[6] I. Janossy, and M. Menyhard, Surf. Sci. 25, 647 (1974)

[7] F. Bart, and M. Gautier, Surf. Sci. Lett. 311, L671 (1994)

[8] J. P. Bachheimer, and G. Dolino, Phys. Rev. B 11, 3185 (1975)

[9] N. H. de Leeuw, F. M. Higgins, and S. C. Parker, J. Phys. Chem. B 103, 1270 (1999)

[10] G. M. Rignanese, A. D. Vita, J. C. Charlier, X. Gonze, and R. Car, Phys. Rev. B 61, 13250 (2000)

[11] P. Naël,T. Sarlat, E. Søndergård, A. Apfolter, and B. Holst, to be submitted to Appl. Phys Letters.

[12] A. Apfolter, Diploma Thesis, Graz University of Technology 2005

[13] C. S. Lent, and P. I. Cohen, Surf. Sci. 139, 121 (1984)

[14] A. J. Gratz, and P. Bird, Geochem. et Cosmochim. 57, 977 (1992)

[15] A.J. Gratz, S. Manne, and P.K Hansma, Science, 251, 1343 (1991)

[16] G. Wolken, J. Chem. Phys. 58, 3047 (1973) 